frequency and airway inflammation have been suggested. There is however limited data describing the reproducibility of sputum microbiology results in clinically stable COPD patients.

Aims Examine repeatability of sputum microbiology in subjects with stable COPD over time.

Methods Subjects with COPD were enrolled into an observational study and seen at baseline and at stable visits after 3 and 6 months. Sputum was obtained and samples were divided and analysed over time using standard culture, semi-quantitative bacterial count (colony forming units, CFU), PCR for potentially pathogenic organisms [(Haemophilus influenzae (HI), Streptococcus pneumoniae (SP), Staphylococcus aureus (SA), Moraxella catarrhalis (MC)] and quantitative bacterial $16 \mathrm{~S}$ analysis.

Results 63 subjects provided paired sputum samples; 52 were male with a mean (SD) FEV1 (L) and FEV1/FVC ratio (\%) of 1.48(0.54) and $53 \%(12.8)$ respectively. $40 \%$ were current smokers with an exacerbation frequency of 3 in the preceding year.

Results for standard culture were divided into two groups (culture positive or negative). Results are expressed as Kappa values (95\% $\mathrm{CI})$. There was moderate agreement after 3 months, Kappa $=0.48$ (0.24 to 0.71$)$; and after 6 months, Kappa $=0.50$ (0.25 to 0.76 ). Individual PCR revealed fair agreement after both time intervals. After 3 months, $\mathrm{HI}=0.17(-0.08$ to 0.43$), \mathrm{SA}=0.27(-0.03$ to 0.56$)$, $\mathrm{SP}=0.30(0.06$ to 0.53$), \mathrm{MC}=0.19(-0.04$ to 0.43$)$. After 6 months, $\mathrm{HI}=0.09$ ( -0.18 to 0.35$), \mathrm{SA}=0.10(-0.22$ to 0.43$), \mathrm{SP}=0.37(0.13$ to $0.62)$ and $\mathrm{MC}=-0.14(-0.4$ to 0.11$)$.

Quantitative bacterial analysis demonstrated no differences (mean difference; $95 \% \mathrm{CI}$ ) at 3 or 6 months in bacterial load measured by CFU ( $-0.18 ;-0.41$ to $0.04, p=0.11$ and $-0.06 ;-0.32$ to 0.2 , $\mathrm{p}=0.65$ respectively) or $16 \mathrm{~S}(-0.03 ;-0.28$ to $0.33, \mathrm{p}=0.86$ and -0.1 ; -0.42 to $0.22, p=0.54$ respectively).

Discussions These results demonstrate that sputum microbiological assessment in stable COPD is complex. Further longitudinal assessments of sputum microbiology and associations with clinical features are needed.

\section{P213 THE PREVALENCE AND IMPACT OF GASTRO-OESOPHAGEAL REFLUX SYMPTOMS IN STABLE COPD PATIENTS}

doi:10.1136/thoraxjnl-2012-202678.274

ARC Patel, BS Kowlessar, GC Donaldson, R Singh, AJ Mackay, S Brill, JA Wedzicha, JR Hurst. Centre for Respiratory Medicine, UCL Medical School, London, UK

Introduction Gastro-oesophageal reflux disease (GORD) has been associated with increased COPD exacerbation frequency (Terada et al, Thorax 2008) and was independently associated with the frequent exacerbator phenotype in the ECLIPSE study (Hurst et al, NEJM 2010). We aimed to quantify the prevalence and impact of GORD in stable COPD in terms of airflow limitation, dyspnoea, health status and exacerbation frequency in a well-characterised cohort.

Methods Stable outpatients from the London COPD cohort completed the Frequency Scale for the Symptoms of Gastro-oesophageal reflux (FSSG), Hull Airway Reflux Questionnaire (HARQ), MRC dyspnoea score, and St George's Respiratory Questionnaire (SGRQ) during clinic visits. Spirometry was performed in accordance with ATS/ERS guidance. Comorbidities including GOR and all medications were recorded by clinical research staff. Exacerbations were defined using our usual symptomatic criteria from daily diary cards (Seemungal et al, AJRCCM 1998).

Results 156 stable COPD patients had a mean \pm SD age of $72.2 \pm 9.7$ years, $63 \%$ male, $29 \%$ current smokers, median (IOR) $46(25,59)$ pack years, mean \pm SD FEV1 $1.34 \pm 0.57 \mathrm{~L}$ and $54.4 \pm 20.2 \%$ predicted, BMI $26.5 \pm 5.7 \mathrm{~kg} / \mathrm{m}^{2}$.

$60(38 \%)$ patients had a diagnosis of GORD, of whom $45(75 \%)$ were taking regular acid suppression therapy (42 proton pump inhibitors, $3 \mathrm{H}_{2}$ antagonists).
COPD patients with GORD had a higher exacerbation frequency than those without (median (IOR) $2.0(1.0,3.4)$ vs $1.2(0.0,3.0)$ per year, $p=0.028$ ). In those with GORD, the use of acid suppression therapy did not impact exacerbation frequency (median (IOR) 2.0 $(1.0,4.0)(n=45)$ vs $2.0(1.0,3.0)(n=15)$ per year, $p=0.431)$.

FSSG and HARQ scores were both related to COPD exacerbation frequency (rho $=0.159, p=0.050$ and rho $=0.185, p=0.021$ respectively) and more strongly to SGRQ $(r=0.425, p<0.001$ and $r=0.439$, $\mathrm{p}<0.001$ respectively). They were not related to MRC dyspnoea score ( $r h o=0.079, p=0.367$ and $r h o=0.126, p=0.148$ respectively) or FEV1\% predicted $(r=-0.063, p=0.452$ and $r=-0.067, p=0.416$ respectively).

Conclusions GORD is common in COPD and is associated with higher exacerbation frequency, although acid suppression therapy does not appear to affect this. Higher GORD symptom scores relate to worse health status and higher exacerbation frequency but not to airflow limitation or dyspnoea. Understanding the mechanisms may lead to novel effective interventions in COPD.
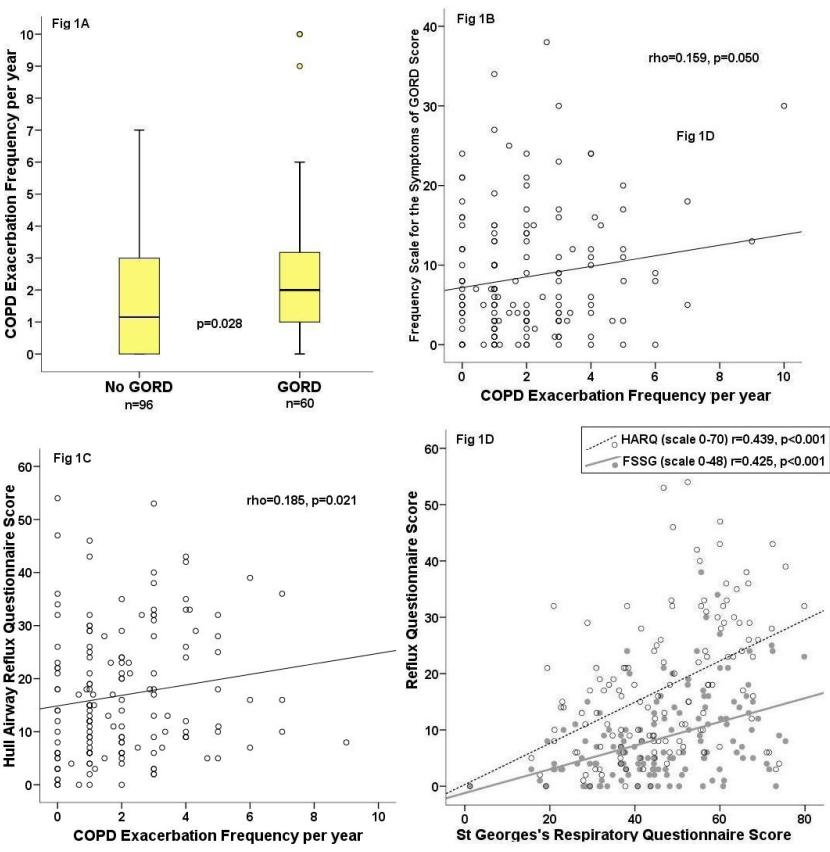

Abstract P213 Figure 1

\section{P214 GASTRO-OESOPHAGEAL REFLUX SYMPTOMS DURING COPD EXACERBATIONS}

doi:10.1136/thoraxjnl-2012-202678.275

ARC Patel, BS Kowlessar, GC Donaldson, AJ Mackay, R Singh, S Brill, JA Wedzicha, JR Hurst. Centre for Respiratory Medicine, UCL Medical School, London, UK

Introduction Gastro-oesophageal reflux disease (GORD) has been associated with increased COPD exacerbation frequency (Terada et al, Thorax 2008) and was independently associated with the frequent exacerbator phenotype in the ECLIPSE study (Hurst et al, NEJM 2010). We aimed to quantify any changes in GORD symptoms during COPD exacerbations.

Methods Outpatients from the London COPD cohort completed the Frequency Scale for the Symptoms of Gastro-oesophageal reflux (FSSG) and Hull Airway Reflux Questionnaire (HARQ) during stable-state clinic visits and at exacerbation, within a week of symptom-onset, and prior to systemic therapy. FSSG and HARO scores range from 0-48 and 0-70 respectively, with significant reflux thought to be associated with scores of $\geq 8$ and $\geq 13$ respectively. 
Exacerbations were defined using our usual symptomatic criteria from daily diary cards (Seemungal et al, AJRCCM 1998).

Results 42 COPD patients had a mean \pm SD age of $72.7 \pm 8.7$ years, $64 \%$ male, $17 \%$ current smokers, median(IOR) 46 (19.71) pack years, mean \pm SD stable FEV1 $1.22 \pm 0.64 \mathrm{~L}$ and $50.7 \pm 21.5 \%$ predicted, BMI $27.7 \pm 7.4 \mathrm{~kg} / \mathrm{m} 2.13$ (31\%) patients had a diagnosis of GORD, of whom 12 (92\%) were taking regular acid suppression therapy. There was a median (IOR) interval of $141(80,233)$ days between the stable and exacerbation visits.

Although median (IOR) FSSG and HARO scores were higher at exacerbation compared to the stable state, this was not statistically significant due to high variability $(6.5(4.0,13.0)$ vs $5.0(1.5,11.5)$, $p=0.247$ and $15.5(9.0,23)$ vs $18.5 \quad(11.5,24.5), p=0.096$ respectively).

$16 / 42(38 \%)$ patients had a high FSSG score $(\geq 8)$ in the stable state compared with $20 / 42(48 \%)$ at exacerbation $(p=0.378) .10 / 42$ (24\%) changed from a low stable FSSG to a high score at exacerbation.

$27 / 42(64 \%)$ patients had a high HARQ score $(\geq 13)$ in the stable state compared with $30 / 42(71 \%)$ at exacerbation $(p=0.483) .7 / 42$ $(17 \%)$ changed from a low stable HARQ to a high score at exacerbation.

Conclusions GORD symptom scores are not significantly higher during acute COPD exacerbations. Due to high variability, approximately one fifth of COPD patients had a low GORD symptom score when stable and high scores at exacerbation implicating worsening reflux in some exacerbations. Further work is required to understand the potential mechanisms.
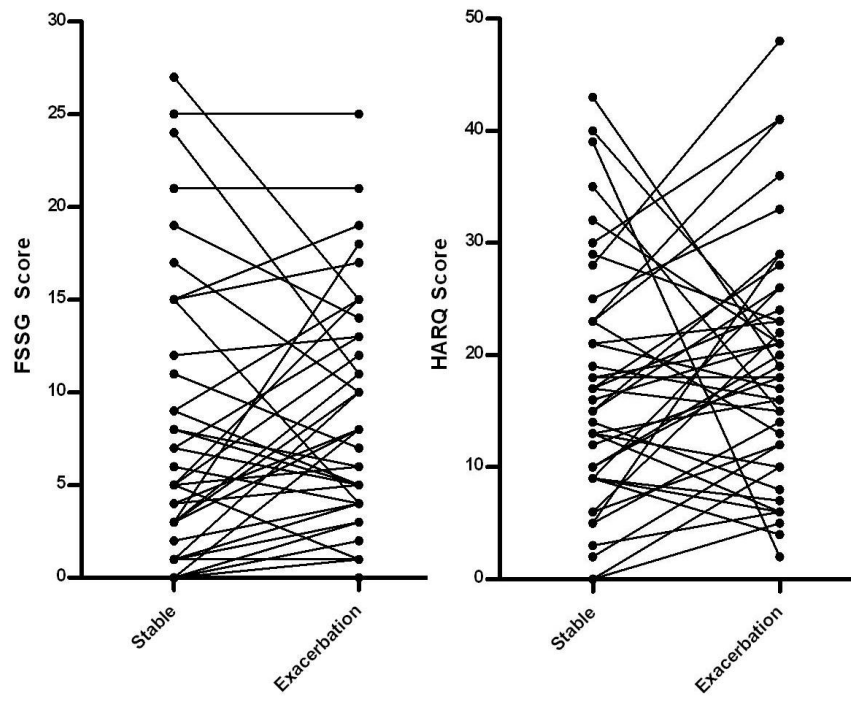

Abstract P214 Figure 1

\section{P215 LEFT VENTRICULAR HYPERTROPHY IN CHRONIC OBSTRUCTIVE PULMONARY DISEASE WITHOUT HYPOXAEMIA: THE ELEPHANT IN THE ROOM?}

doi:10.1136/thoraxjnl-2012-202678.276

WJ Anderson, BJ Lipworth, S Rekhraj, AD Struthers, J George. University of Dundee, Dundee, United Kingdom

Background Chronic obstructive pulmonary disease (COPD) is associated with significant cardiovascular mortality. Left ventricular hypertrophy $(\mathrm{LVH})$ is a pivotal cardiovascular risk factor. The prevalence of LVH in COPD is currently unknown.

Methods We performed a pilot study of 93 normoxaemic COPD patients and 34 controls. Patients underwent echocardiography to measure left ventricular (LV) dimensions; electrocardiography; 24-hour blood pressure (BP) recording; and serum B-type natriuretic peptide (BNP) levels, along with spirometry and oxygen saturations. Results COPD patients' oxygen saturations were normal at $96.5 \%$ (95\%CI: $96.1-97.0 \%)$, with a mean FEV1 of $70.0 \%$ predicted $(95 \%$ CI: $65.2-74.8 \%$ ). $30.1 \%$ of COPD patients met echocardiographic criteria for LVH based on LV mass index, with more LVH in females than males ( $43.2 \%$ vs. $21.4 \%$, p=0.02). LV mass index in COPD was $96.2 \mathrm{~g} / \mathrm{m}^{2}$ (95\%CI: $\left.90.1-102.7 \mathrm{~g} / \mathrm{m}^{2}\right)$ vs. controls $82.9 \mathrm{~g} / \mathrm{m}^{2}(95 \% \mathrm{CI}$ : $75.8-90.6 \mathrm{~g} / \mathrm{m}^{2}$ ), $\mathrm{p}=0.017$ (Figure 1). LV mass index remained high in COPD patients in the absence of hypertension history $\left(94.5 \mathrm{~g} / \mathrm{m}^{2} \mathrm{vs}\right.$. $\left.79.9 \mathrm{~g} / \mathrm{m}^{2}, \mathrm{p}=0.015\right)$ and with $24-\mathrm{hr}$ systolic $\mathrm{BP}<135 \mathrm{mmHg}(96.7 \mathrm{~g} /$ $\mathrm{m}^{2}$ vs. $\left.82.5 \mathrm{~g} / \mathrm{m}^{2}, \mathrm{p}=0.024\right)$. LV ejection fraction (mean $=63.4 \%$ ) and BNP $($ mean $=28.7 \mathrm{pg} / \mathrm{ml}$ ) were normal in COPD patients. Mean $24 \mathrm{hr}$ BP was normal in COPD patients at $125 / 72 \mathrm{mmHg}$. Electrocardiography was less sensitive for detecting LVH than echocardiography.

Conclusion LVH was present in a significant proportion of normotensive, normoxaemic COPD patients, especially in females, along with normal LV ejection fraction and BNP levels. Clinical trials are therefore indicated to evaluate treatments to regress $\mathrm{LVH}$ in patients with COPD.

\section{Figure 1}

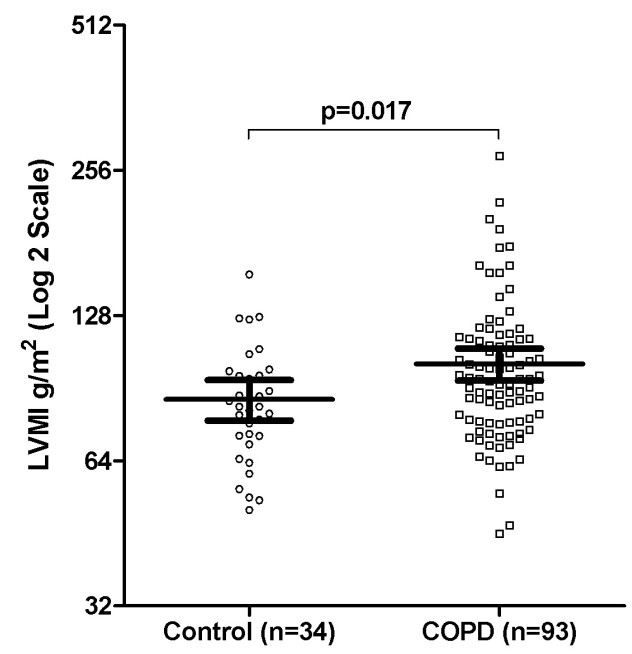

Left Ventricular Mass Index (LVMI) between groups. Presented as scatterplots of all data points with solid lines representing respective geometric means and $95 \% \mathrm{Cls}$. Means comparison with Student's T-test for unpaired samples (two-tailed). Significance set at $p<0.05$.

Abstract P215 Figure 1

\section{P216 IMPACT OF TACHYCARDIA AND NEW ONSET ATRIAL FIBRILLATION IN ACUTE EXACERBATIONS OF COPD}

doi:10.1136/thoraxjnl-2012-202678.277

'PM Short, ${ }^{1} J D$ Chalmers, ${ }^{2} \mathrm{AR}$ Akram, ${ }^{3} \mathrm{~A}$ Singanayagam, ' $\mathrm{S}$ Schembri, ${ }^{1} \mathrm{PA}$ Williamson. ${ }^{1}$ Ninewells Hospital, Dundee, United Kingdom; ${ }^{2}$ Royal Infirmary of Edinburgh, Edinburgh, United Kingdom; ${ }^{3}$ Imperial College, London, United Kingdom

Introduction and Objectives Cardiac arrhythmias are regularly found in patients with COPD, with higher frequencies reported during exacerbations. We wished to examine the impact of tachycardia and new onset atrial fibrillation (AF) on long term outcome in patients hospitalised with a COPD exacerbation. 